

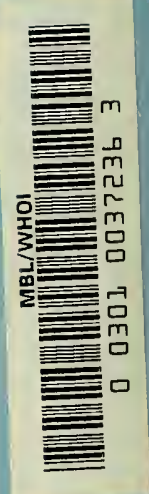




\section{PRESSURE FLUCTUATIONS ON THE WALL ADJACENT TO A TURBULENT BOUNDARY LAYER}

$$
\text { by }
$$

Mark Harrison, Ph. D. 


\section{TABLE OF CONTENTS}

Page

ABSTRACT ............................. . . . . 1

INTRODUCTION . . . . . . . . . . . . . . . . . . . . . . . 1

EXPERIMENTL APPARATUS . . . . . . . . . . . . . . . . . . . 2

The Wind Tunnel . . . . . . . . . . . . . . . . . . . 2

The Pressure Transducer . . . . . . . . . . . . . . . . . . . . 3

Electrical Equipment . . . . . . . . . . . . . . . . . . . . . 4

EXPERIMENTAL RESULTS . . . . . . . . . . . . . . . . . . . . 5

Spectral Density of the Pressure Fluctuations . . . . . . . . . . . . . . 5

Longitudinal Cross Spectral Density . . . . . . . . . . . . . . . . 7

THE TRANSVFRSE CORRELATION . . . . . . . . . . . . . . . . . . . 12

REFERENCES . . . . . . . . . . . . . . . . . . . . . 12

\section{LIST OF ILLUSTRATIONS}

Page

Figure 1 - Schematic of Adaptation of the Altec 21-BR-180 Microphone for Spectral Density Measurements . . . . . . . . . . . . . . . 3

Figure 2 - Schematic of Microphone Adapted for Cross Spectral and Correlation Measurements . . . . . . . . . . . . . . . . 3

Figure 3 - Schematic Diagram of the Equipment Used in the Measurement of the Cross Spectral Density . . . . . . . . . . . . . . . . . . . 4

Figure 4 - Spectral Density of the Pressure Fluctuations . . . . . . . . . . . . 6

Figure 5 - Real and Imaginary Parts of the Longitudinal Cross Spectral Density. • • 9

Figure 6 - Longitudinal Coherence of the Pressure Fluctuations . . . . . . . . . 11

Figure 7 - Transverse Correlation Function . . . . . . . . . . . . . . . . . 12 


\section{ABSTRACT}

A fully developed turbulent boundary layer with a zero pressure gradient has been studied in a subsonic wind tunnel. The pressure fluctuations on the wall of the wind tunnel were measured by small flush-mounted microphones. Data for the dimensionless spectral density of the pressure fluctuations, $P(f) / \rho^{2} U_{0}^{3} \delta^{*}$, as a function of the frequency parameter $f \delta^{*} / U_{0}$ are presented; $P(f), \rho, U_{0}, \delta^{*}$, and $f$ are respectively the spectral density, fluid density, free stream velocity, boundary layer displacement thickness, and frequency. Data for the coefficient $\left(\overline{p^{2}}\right)^{1 / 2} / 1 / 2 \rho U_{0}^{2}$ are also presented. The transverse cross correlation for the pressure fluctuations was studied by using two flush mounted microphones. Rather than the longitudinal cross correlation, the longitudinal cross spectral density was studied, yielding a measure of the coherence of the pressure fluctuations at two points as a function of $f x / U_{c}$, where $x$ is the longitudinal spacing of the two points and $U_{c}$ is the effective convection velocity of the spatial pattern.

\section{INTRODUCTION}

Pressure fluctuations necessarily coexist with velocity fluctuations in a turbulent flow. For incompressible flow the pressure and velocity are related by the equation

$$
\nabla^{2} p=-\rho \frac{\partial^{2} u_{i} u_{j}}{\partial x_{i} \partial x_{j}}
$$

which can be obtained by combining the continuity and the Navier-Stokes equations. The solution to this equation is given in terms of an intagration over all space and shows that for the case of boundary-layer flow, the vanishing of the velocity fluctuation at the wall does not imply the vanishing of the pressure fluctuations at the wall.

It is these pressure fluctuations on the wall adjacent to the turbulent boundary layer that are the focus of interest in this study. Some measurements of the characteristics of these pressure fluctuations are reported and the meaning of the measurements are interpreted.

There are several reasons for being interested in the characteristics of the pressure fluctuations. A direct and immediate need exists for data in the field of noise due to unsteady flow. When a turbulent boundary layer develops on a flexible wall, the motion of the wall which results from the pressure fluctuations generates a sound field. Some of the noise in aircraft has been attributed to this mechanism. Kraichnan ${ }^{1,2}$ has given a theoretical

\footnotetext{
${ }^{1}$ References are listed on page 12 .
} 
discussion of this problem which begins with the interpretation of Equation [1] for the case of boundary-layer flow.

There are also fundamental reasons for being interested in the pressure fluctuations associated with a turbulent boundary layer. There is considerable uncertainty as to how energy is produced, convected, diffused, and dissipated in the turbulent boundary layer. In particular, the role of pressure fluctuations is but vaguely understood. Although this work does not measure the pressure fluctuations inside the boundary layer, it does constitute a start on this very challenging problem and it is believed that a further extension of this work will prove valuable.

To obtain the data for this report, the boundary layer on the wall of a subsonic wind tunnel was studied. Pressure transducers with a very small active area were mounted flush with the wall. Using conventional sound analysis equipment consisting of a narrow tunable filter and an rms meter, it was possible to obtain the spectral density ${ }^{*}$ of the pressure fluctuations at a point on the wall.

By measuring the pressure fluctuations in the total frequency range, it was possible to obtain the mean square value for the pressure fluctuations. This value was also checked by integrating over the spectral density.

By using two pressure transducers and studying the correlation and the cross spectral density ${ }^{*}$ between the pressure fluctuations at the two points, it was possible to study the spatial pattern of the pressure fluctuations and how rapidly it evolves as it is convected downstream. By measuring the time delay necessary to maximize the correlation between the two points it was possible to measure the velocity at which the pattern was convected.

These, and other results, are presented and discussed.

\section{EXPERIMENTAL APPARATUS}

\section{THE WIND TUNNEL}

The wind tunnel was a closed circuit subsonic tunnel. The velocity range of the tunnel was from 50 to $200 \mathrm{ft} / \mathrm{sec}$. The transverse dimensions of the working section were 20 by 15 inches. The measurements were performed on the wall of the working section, 5 feet downstream from the entrance nozzle. At this point the displacement thickness of the boundary layer was 0.105 inch at a velocity of $100 \mathrm{ft} / \mathrm{sec}$. By comparing the mean velocity profiles with those given by Klebanoff ${ }^{3}$ it was judged that the boundary layer was nearly well enough developed to show similarity. Unfortunately, the working section was not long enough to permit working further downstream so as to prove that the boundarylayer was exhibiting similarity.

\footnotetext{
* This term will be defined in the section giving the results of the measurements.
} 


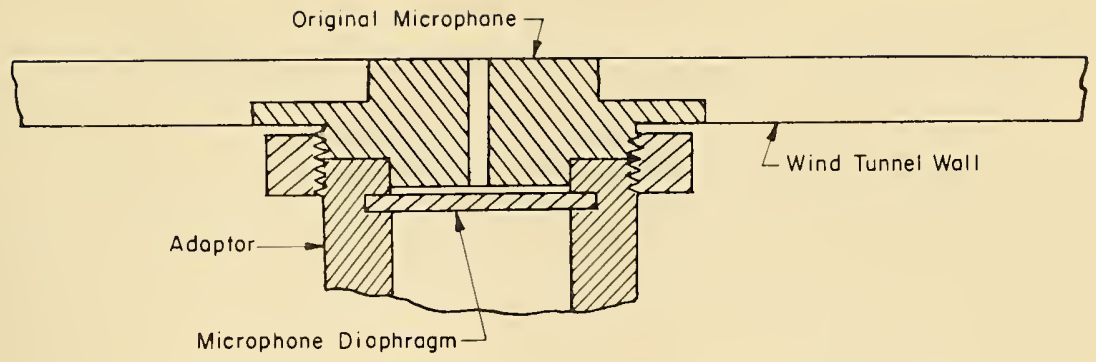

Figure 1 - Schematic of Adaptation of the Altec 21-BR-180 Microphone for Spectral Density Measurements

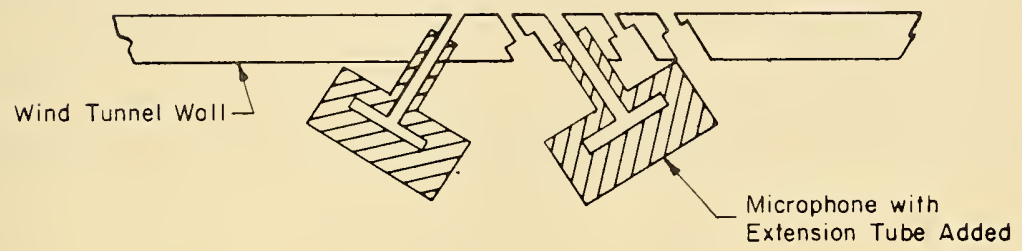

Figure 2 - Schematic of Microphone Adapted for Cross Spectral and Correlation Measurements

\section{THE PRESSURE TRANSDUCER}

The pressure transducer was an adaptation of the Altec 21-3R-180 open-faced microphone. ${ }^{4}$ This microphone was modified as shown in Figure 1 to produce the transducer used for the spectral density measurements. A different modification was required for the correlation measurements. In both cases the upper frequency limit is determined by a resonance between the mass of the air in the orifice and the stiffness of the air in the chamber adjacent to the diaphragm. Well above this resonance frequency the sensitivity is inversely proportional to the frequency range. The resonant response was damped by a cotton plug in the orifice so that some of this frequency range was salvaged.

For the correlation measurements it was necessary to measure at two points separated by a small distance. This necessitated the use of the transducer in the form shown in Figure 2. Unfortunately, this arrangement resulted in a much lower resonant frequency due to increased mass in the tube. Using this transducer, arrangements were made to have a series of holes spaced at 0.125 inch apart in the transverse and longitudinal directions which permitted correlation measurements to be made up to 1.5 inches in 0.125 -inch steps.

The sensitivity of the transducer was $-61 \mathrm{db}$ below 1 volt for $1 \mathrm{dyne} / \mathrm{cm}^{2}$. For the spectral density measurements the usable frequency range was up to $8500 \mathrm{cps}$ and for the 
correlation measurements the usable frequency range was up to $2000 \mathrm{cps}$. The lower limit on the frequency range was imposed by wind tunnel noise and not the transducer.

In order to prove that the active area of the transducer was sufficiently small to insure that the pressure was coherent over its entire face, various size orifices were used. It was found that orifices up to 0.125 inch in diameter were satisfactory for obtaining the data on spectral densities. Smaller orifices were used for the correlation measurements in order to improve the accuracy in the measurement of the separation distance between the transducers.

\section{ELECTRICAL EQUIPMENT}

The spectral density of the pressure fluctuations was obtained by the use of the Muirhead-Pametrada wave analyzer. A frequency band of a nominal 10 percent was used in the analysis. The levels were read on the Ballantine True rms voltmeter. In the section giving the results of the measurements, the details of how to obtain the spectral density from the observed data are given.

The correlation between the pressure fluctuations at two points was measured using rather simple apparatus. The basis for the method used is contained in the algebraic identity

$$
\frac{\overline{e_{1} e_{2}}}{\left(\overline{e_{1}^{2} e_{2}^{2}}\right)^{1 / 2}}=\frac{\overline{\left(e_{1}+e_{2}\right)^{2}}-\overline{\left(e_{1}-e_{2}\right)^{2}}}{4\left(\overline{e_{1}^{2}} \overline{e_{2}^{2}}\right)^{1 / 2}}
$$

where the term on the left is the correlation between $e_{1}$ and $e_{2}$. The bars indicate time averages. $e_{1}$ and $e_{2}$ are here regarded as voltages that are proportioned to the fluctuating pressures $p_{1}(t)$ and $p_{2}(t)$, respectively, where the subscripts denote the two points at which the measurements are performed. With reference to Figure 3, the addition and subtraction of the voltages were accomplished by the transformer. The time averages were accomplished by the Ballantime True rms voltmeter, whose time constant had been increased to two seconds. This produced satisfactory smoothing of the fluctuations. Measuring the correlations is then a matter of making four meter readings, $\left[\overline{\left(e_{1}+e_{2}\right)^{2}}\right]^{1 / 2},\left[\overline{\left(e_{1}-e_{2}\right)^{2}}\right]^{1 / 2},\left(\overline{e_{1}^{2}}\right)^{1 / 2}$, and $\left.\overline{e_{2}^{2}}\right)^{1 / 2}$, and doing the computations indicated by the above algebraic identity.

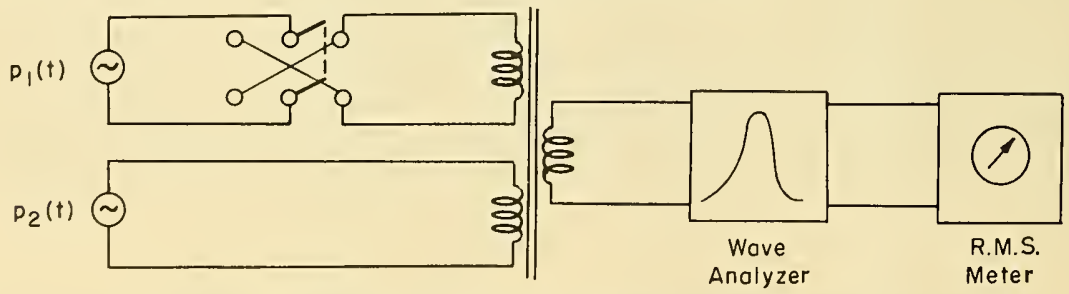

Figure 3 - Schematic Diagram of the Equipment Used in the Measurement of the Cross Spectral Density 
The wave analyzer shown in Figure 3 serves the function of measuring the correlation in a restricted frequency band. As explained later, if observations are made using a sufficiently narrow frequency band, it is possible to use these measurements to obtain the cross spectral density between the pressure fluctuations at two points.

The convection velocity for the partially frozen pattern of the pressure fluctuations was determined by examining the correlation and cross spectral density with time delays. These were produced by a delay line consisting of a multistage low-pass filter. This delay line gave time delays in 40 -microsecond steps up to 6 milliseconds. The delay line had a usable frequency range from zero to a little above $5000 \mathrm{cps}$.

\section{EXPERIMENTAL RESULTS}

\section{SPECTRAL DENSITY OF THE PRESSURE FLUCTUATIONS}

The spectral density of the pressure fluctuations $P(f)$ is defined as 5

$$
P(f)=\lim _{T \rightarrow \infty} \frac{1}{T}\left|A_{T}(f)\right|^{2}
$$

where

$$
A_{T}(f)=\int_{-T}^{T} p(t) e^{-i \omega t} d t
$$

is the Fourier transform of the pressure fluctuations over the time $2 T$.

The basis for the measurement procedure used in obtaining the spectral density begins with the fact that the spectral density and the autocorrelation function of $p(t)$ are Fourier transforms. 5

$$
R(\tau)=\overline{p(t) p(t+\tau)}=\int_{0}^{\infty} P(f) \cos \omega \tau d f
$$

It then follows that

$$
R(0)=\overline{p^{2}}=\int_{0}^{\infty} P(f) d f
$$

According to this equation one can experimentally obtain the spectral density by measuring the mean square output $p_{0}^{2}$ of a narrow tunable filter of band with $\Delta f$ and unity gain. 
That is to say,

$$
P(f)=\lim _{\Delta f \rightarrow 0} \frac{\overline{p_{0}^{2}}}{\Delta f}
$$

This was the procedure used in the work reported here.

It is desirable to present the measured data in dimensionless form. Since the spectral density of the pressure fluctuations has the dimensions of (pressure) ${ }^{2} \times$ time, the dimensionless spectral density was chosen to be $P(f) / \rho^{2} U_{0}^{3} \delta^{*}$ where $\rho, U_{0}$, and $\delta^{*}$ are the fluid density, free steam velocity, and boundary layer displacement thickness, respectively. The frequency $f$ was nondimensionalized as $f \delta^{*} / U_{0}$.

The dimensionless spectral density curves are shown in Figure 4. The data exhibits some lack of similarity over the velocity range studied. As mentioned earlier, the work section of the tunnel was not long enough to permit observations further downstream so

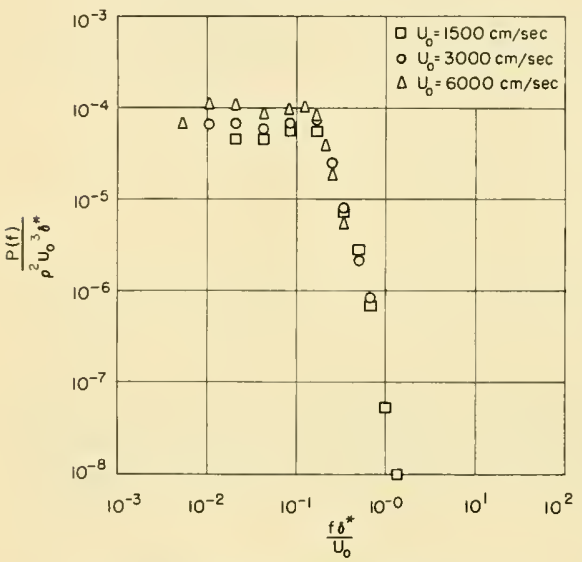

Figure 4 - Spectral Density of the Pressure Fluctuations as to prove that the boundary layer was exhibiting similarity.

The coefficient

$$
K=\frac{\overline{\left(p^{2}\right)^{1 / 2}}}{\frac{1}{2} \rho U_{0}^{2}}
$$

can be obtained either by integrating over the spectral density or by direct measurement of $\overline{p^{2}}$. In view of the lack of similarity exhibited in Figure 4, a definite value cannot be obtained. The values obtained for $K$ have an average value of about $9.5 \times 10^{-3}$ which in excess of the value obtained by Hillmarth. ${ }^{6}$

A surprising feature of the spectral

density is that it does not decrease appreciably at the lowest frequencies measured. For these frequencies, which correspond to wave numbers much smaller than the reciprocal of the characteristic lengths of the boundary layer, Kraichnan has argued that the spectral density should be proportional to the square of the frequency. ${ }^{1}$ There is some evidence that intermittency of the boundary layer has a strong influence on the pressure fluctuations at the lower frequency. Qualitative observations at the lower frequencies revealed an intermittency in the amplitude of the spectral density which could not be accounted for by the randomness of the process. This intermittency gave pressure versus time records not unlike those observed 
by Klebanoff ${ }^{7}$ for the velocity fluctuations in the boundary layer. This type of behavior diminished with increasing frequency and was not noticeable for values of $f \delta^{*} / U_{0}$ exceeding $4 \times 10^{-2}$. Further support for the possibility that intermittency plays a role at the lower frequencies can be found in the fact that at these frequencies, the measurements of the convection velocity yielded a value of about $0.8 U_{0}$. That part of the boundary layer that moves with a mean velocity of $0.8 U_{0}$ is strongly intermittent.

The validity of the above conjectures could be established by repeating this study using fully developed pipe flow.

In view of the preceding, it would have been desirable to have procured data at lower frequencies. Unfortunately, this was not possible due to the tunnel noise which limited the lowest usable frequencies to between 100 and $200 \mathrm{cps}$. The signal-to-noise ratio above this range was always in excess of $20 \mathrm{db}$. This figure was obtained by placing the pressure pickup in the middle of the working section of the tunnel where the flow was essentially laminar. Actually, some noise was undoubtedly induced by the flow around the microphone, so that a better signal-to-noise ratio might actually have existed at the wall.

Vibration pickup by the transducer was not a problem. By simply plugging up the hole in the transducer, the vibration excitation could be measured, which in this case was too low to even consider.

\section{LONGITUDINAL CROSS SPECTRAL DENSITY}

In this work it was decided to study the longitudinal cross spectral density rather than the longitudinal correlation. Since this is a novel approach, a few words of explanation are needed in order to explain the measurement procedure.

The cross spectral density of the pressure fluctuations is defined as

$$
P_{12}(f)=\lim _{T \rightarrow \infty} \frac{1}{T} A_{T}^{*}(f) B_{T}(f)
$$

where the asterisk denotes the complex conjugate, and where

$$
\begin{aligned}
& A_{T}(f)=\int_{-T}^{T} p_{1}(t) e^{-i \omega t} d t \\
& B_{T}(f)=\int_{-T}^{T} p_{2}(t) e^{-i \omega t} d t
\end{aligned}
$$

with the subscripts 1 and 2 denoting the two observation points. 
Using the arguments of Reference 5 which show that the spectral density and the autocorrelation are Fourier transforms, it can be shown that the cross correlation and the cross spectral density are also Fourier transforms:

$$
R_{12}(\tau)=\overline{p_{1}(t) p_{2}(t+\tau)}=\frac{1}{2} \int_{-\infty}^{\infty} P_{12}(f) e^{i \omega t} d f
$$

Since $P_{12}(f)$ is complex, we can write

$$
P_{12}(f)=U_{12}(f)+i V_{12}(f)
$$

where it follows from the definition of $P_{12}(f)$ that the real functions $U_{12}(f)$ and $V_{12}(f)$ are even and odd, respectively. Accordingly, we can write

$$
R_{12}(\tau)=\int_{0}^{\infty} U_{12}(f) \cos \omega \tau d f-\int_{0}^{\infty} V_{12}(f) \sin \omega \tau d f
$$

This last equation provides the basis for an experimental method of measuring the real and imaginary parts of the cross spectral density. If the cross correlation is measured with zero time delay, then

$$
R_{12}(0)=\int_{0}^{\infty} U_{12}(f) d f
$$

If the cross correlation is measured in a narrow band of frequencies $\Delta f$ as shown in Figure 3 , then

$$
U_{12}(f)=\frac{f^{R_{12}}(0)}{\Delta f}
$$

where $f_{12}(0)$ denotes the cross correlation measured in the narrow frequency band. The real part of the normalized cross spectral density $u_{12}(f)$ is then

$$
u_{12}(f)=\frac{U_{12}(f)}{\left[P_{1}(f) P_{2}(f)\right]^{1 / 2}}
$$


Similarly, $V_{12}(f)$ is obtained by using a time delay

$$
\tau_{0}=\frac{1}{4 f_{0}}
$$

where $f_{0}$ is here the midband frequency of the narrow filter. The imaginary part of the normalized cross spectral density $v_{12}(f)$ is

$$
v_{12}(f)=\frac{V_{12}(f)}{\left[P_{1}(f) P_{2}(f)\right]^{1 / 2}}
$$

It is noted that the measurement of $u_{12}(f)$ and $v_{12}(f)$ yields more information than the more commonly measured normalized cross correlation since the normalized cross correlation may be obtained by integration.

For this work $P_{1}(f)=P_{2}(f)$ since the two measurement points are sufficiently close together that the boundary layer has not changed in character.

The real and imaginary parts of the cross spectral density as a function of $f_{0} x / 0.8 U_{0}$ are shown in Figure 5. The distance between the two measurement points has been designated as $x$, and the midband frequency of the narrow filter has been designated as $f_{0}$. The factor of 0.8 will now be accounted for.

By measuring the cross correlation in a narrow band of frequencies, and by determining the time delay $T$ that maximized this correlation, an effective convection velocity $U_{c}=x / T$ of the spectral pattern was determined. For all frequencies up to $2000 \mathrm{cps}$ (the upper limit

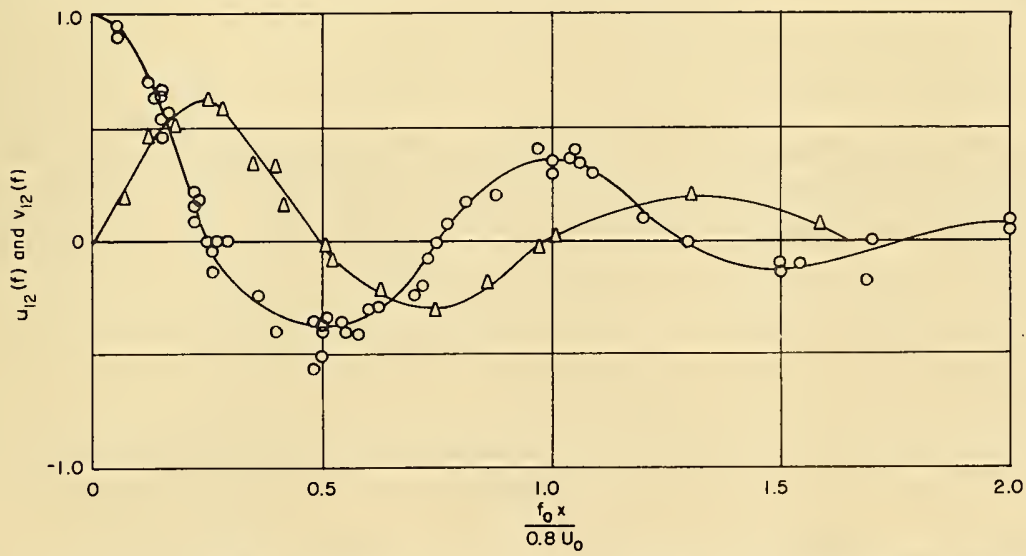

Figure 5 - Real and Imaginary Parts of the Longitudinal Cross Spectral Density 
of the transducer for this circumstance), it was found that the convection velocity was about $0 . S U_{0}$. It was expected that the convection velocity would decrease with increasing values of frequency as a consequence of the quadripole nature of the right hand term in Equation [1]. The measurement procedure was not sufficiently precise to reveal such a relationship even though there is reasonable certainty of its existence.

It can be seen that the spatial pattern of the pressure fluctuations is not convected downstream as though it is frozen. If this were so, the real part of the normalized cross spectral density can be shown by a simple computation, for the case of a filter with a rectangular frequency response, to be

$$
u_{12}\left(\frac{f_{0} x}{0.8 U_{0}}\right)=\frac{\sin \left(\frac{2 \pi\lrcorner f x}{0.8 U_{0}}\right)}{\left(\frac{2 \pi \Delta f x}{0.8 U_{0}}\right)} \cos \left(\frac{2 \pi f_{0} x}{0.8 U_{0}}\right)
$$

where the first factor on the right is an effect due to the finite bandwidth $\Delta f$. Since, in these measurements a constant 10 percent bandwidth was used, this factor is negligibly different from unity.

The departure, for the real part of the data of Figure 5 , from $\cos 2 \pi f_{0} x / 0.8 U_{0}$ is a measure of the development of an uncorrelated component in the pressure fluctuations during its travel from the upstream to the downstream measurement point.

A measure for the uncorrel ated component can be formulated mathematically. The downstream pressure fluctuation $p_{2}(t)$ can be resolved into three components whose cross spectral densities with the upstream pressure fluctuation are, respectively, real, imaginary, and zero. That is,

$$
p_{2}(t)=\alpha(t)+\beta(t)+\gamma(t)
$$

where $P_{1 \alpha}=U_{12}, P_{1 \beta}=i V_{12}, P_{1 \gamma}=0$. The vanishing of the spectral density is equivalent to saying that the correlation for $p_{1}(t)$ and $\gamma(t)$ is zero. This resolution can be affected using the definition for cross spectral density. The spectral densities for $\alpha(t), \beta(t)$, and $\gamma(t)$ are

$$
\begin{aligned}
& P_{\alpha}=U_{12}^{2} / P_{1} \\
& P_{\beta}=V_{12}{ }^{2} / P_{1} \\
& P_{\gamma}=P_{2}-\left|P_{12}\right|^{2 / P_{1}}
\end{aligned}
$$




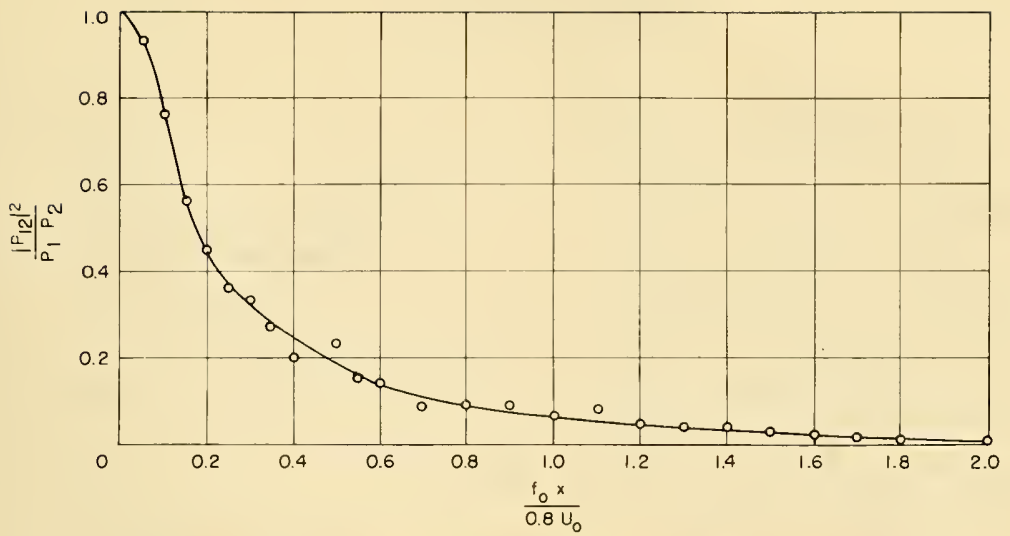

Figure 6 - Longitudinal Coherence of the Pressure Fluctuations

so that

$$
\overline{\gamma^{2}(t)}=\int_{0}^{\infty} P_{\gamma}(f) d f
$$

and can be computed in terms of the measured data $P_{1}(f), P_{2}(f)$, and $\left|P_{12}\right|^{2}=U_{12}{ }^{2}+V_{12}{ }^{2}$. Perhaps of greater interest is the quantity

$$
\frac{\left|P_{12}\right|^{2}}{P_{1} P_{2}}=1-\frac{P_{\gamma}}{P_{2}}
$$

which can be taken as a measure of the coherence of $p_{2}(t)$ with $p_{1}(t)$. This quantity is plotted in Figure 6.

There are several important limitations in the data presented in Figure 5. One trend in the data that is desirable to investigate is the effect of the parameter $f \delta^{*} / U_{0}$. Unfortunately, the transducer used for the cross spectral density measurements had a usable frequency range up to only $2000 \mathrm{cps}$. Consequently, only the flat part of the spectral density, as shown in Figure 4, could be investigated. The upper limit of $2000 \mathrm{cps}$ set an upper limit on $f \delta^{*} / U_{0}$ between 0.083 and 0.33 . As can be seen in Figure 4, this does not permit investigation of the cross spectral density of the pressure fluctuations in the range where the spectral density is rapidly decreasing with frequency. With reference to the velocity fluctuations in the boundary layer, the region investigated corresponded to the region where the velocity spectral density is described by the $-5 / 3$ law where inertia forces dominate. It would be interesting to procure data for the cross spectral density in the range where viscous forces dominate. 


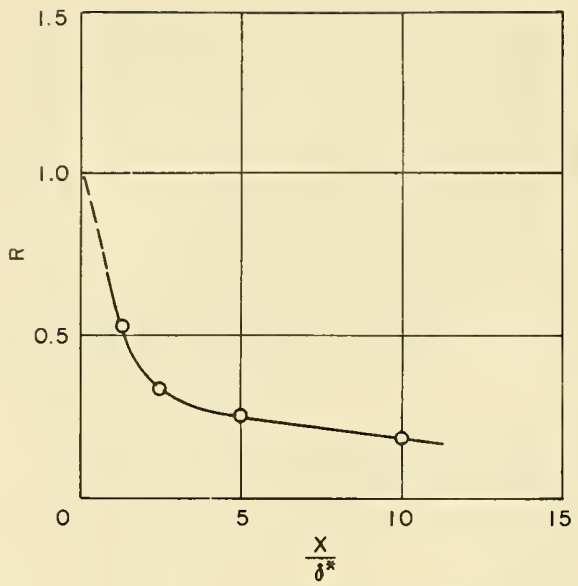

Figure 7 - Transverse Correlation Function

\section{THE TRANSVERSE CORRELATION}

The transverse correlation was measured using the equipment of Figure 3 with the wave analyzer omitted. The data are presented in Figure 7. Unfortunately, these data have not been checked carefully since they were taken on the last day the wind tunnel was available for this work. Since we are not concerned here with the convection of a nearly frozen pattern, the cross spectral densities were not investigated. For the purpose of a cross check, however, it would have been valuable to have obtained these data. Another limitation of the data is that its variation of speed was not checked because of limitations on experimental time.

\section{REFERENCES}

1. Kraichnan, R.H., Journal Acoustical Society of America, Vol. 28, p. 378 (1956).

2. Kraichnan, R.H., Journal Acoustical Society of America, Vol. 29, p. 65 (1957).

3. Klebanoff, P.S. and Diehl, Z.W., National Advisory Committee for Aeronautics Report 1110 (1952).

4. Hilliard, J.K. and Fiala, W.T., Journal Acoustical Society of America, Vol. 29, p. 254 (1957).

5. James, Nichols, and Phillips, "Theory of Servomechanisms," McGraw-Hill Book Company, Inc., New York (1947) Chapter 6.

6. Willmarth, W.W., Journal Acoustical Society of America, Vol. 28, p. 1048 (1956).

7. Klebanoff, P.S., National Advisory Committee for Aeronautics Report 1247 (1955). 


\section{INITIAL DISTRIBUTION}

\section{Copies}

11 CHBUSHIPS. Library (Code 312)

5 Tech Library

1 Tech Asst to Chief (Code 106)

1 Applied Science (Code 370)

1 Prelim Des (Code 420)

1 Sonar Systems (Code 454)

1 Submarines (Code 525)

1 Torpedo Countermeasures (Code 631 M)

2 CHBUAER, Aero \& Hydro Br (DE-3)

2 CHBUORD, Underwater Ordnance (Re 6)

3 CHONR

2 Fluid Mech $\mathrm{Br}$ (Code 438)

1 Undersea Warfare $\mathrm{Br}$ (Code 466)

2 CDR, USNOL

2 CDR, USNOTS, Pasadena Annex, Calif.

2 CO \& DIR, USNUSL, New London, Conn.

2 CO \& DIR, USNEL, San Diego, Calif.

1 CO, USNATC, Philadelphia, $P$ a.

2 DIR, USNRL

1 DIR, Ballistic Res Lab, Aberdeen Prov Grd, Aberdeen, Md.

2 Asst Secy (R \& E)

10 ASTIA, Arlington, $V a$.

2 Dir, NASA

1 Dir, Langley Res Ctr, Langley Field, Va. Attn: Mr. Harvey H. Hubbard

2 Dir, Ames Res Ctr, Moffett Field, Calif.

2 Dir, Lewis Res Ctr, Cleveland, 0.

2 Headquarters, Air Materiel Command, Wright Air Ctr, Wright-Patterson AFB, 0 .

1 Mr. Donald H. Eldredge

2 Dir, Natl BuStand

1 Dr. G.B. Schubauer

2 Acoustics Res Lab, Harvard University, Cambridge, Mass.
Copies

2 Penn St Univ, University Park, $\mathrm{Pa}$. 1 Dir, ORL

1 Dept of Physics, Brown Univ, Providence, R.I.

1 Dir, Daniel Guggenheim Aero Lab, CIT, Pasadena, Calif.

2 Dr. H. Rouse, Dir, lowa Inst of Hydra Res, St Univ of lowa, lowa City, la.

2 Dir, Hydro Lab, CIT, Pasadena, Calif.

1 Dir, Armour Res Foundation, Chicago, III.

1 Dir, Woods Hole Oceanographic Inst, Woods Hole, Mass.

1 Dir, Hudson Lab, Dobbs Ferry, N.Y.

1 Librarian, American Inst of Physics, New York, N.Y.

1 Librarian, ASME, New York, N.Y.

1 Librarian, Inst of the Aero Sci, New York, N.Y.

2 Editor, Appl Mech Reviews, Southwest Res Inst, San Antonio, Tex.

1 Editor, Aero Engin Review, New York, N.Y.

1 Prof. Alan Powell, Dept of Aero, Univ of Calif, Los Angeles, Calif.

1 Dr. R.H. Kraichnan, Inst of Math Sci, New York Univ, New York, N.Y.

2 Inst of Aerophysics, Univ of Toronto, Toronto, Canada

9 ONR Br Office, London, England 



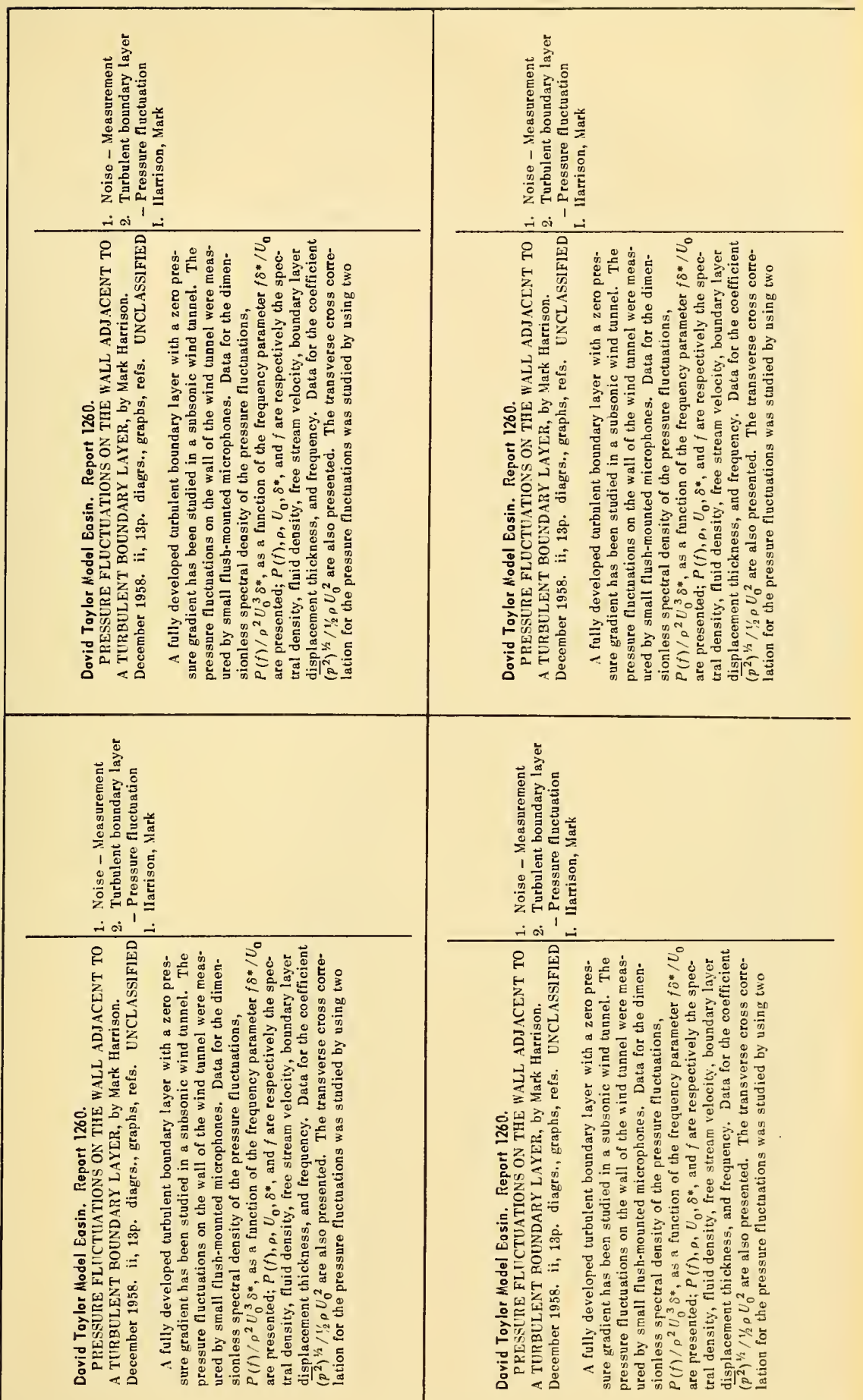



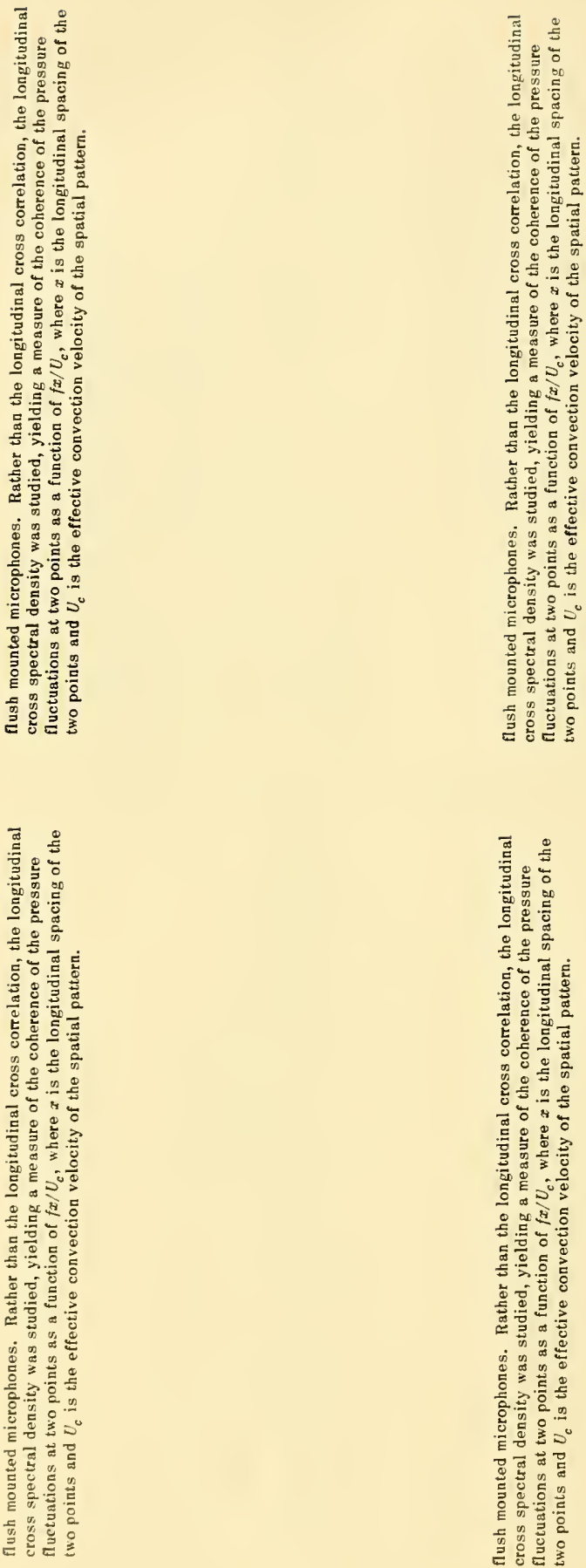


\begin{tabular}{|c|c|}
\hline 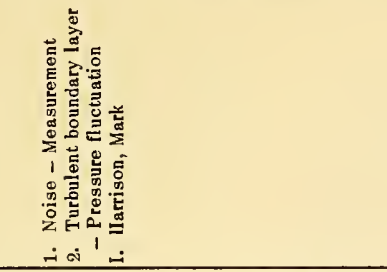 & 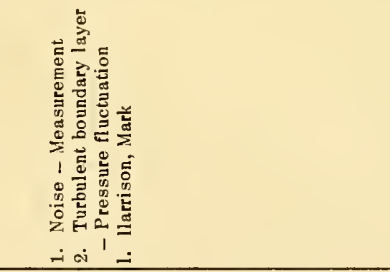 \\
\hline 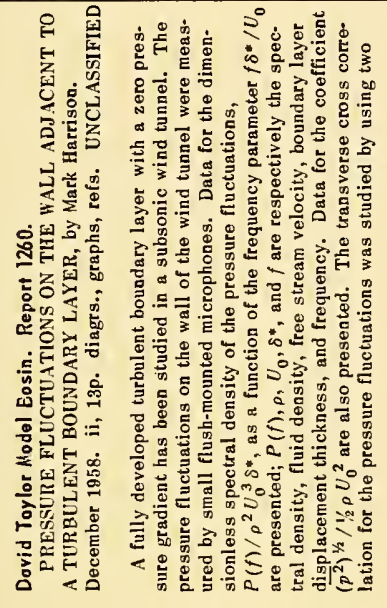 & 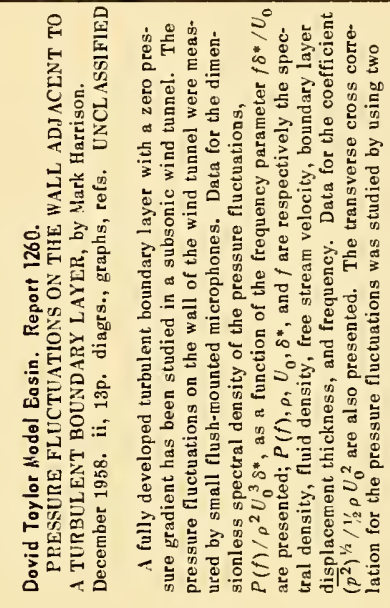 \\
\hline 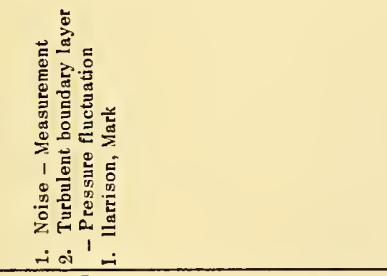 & 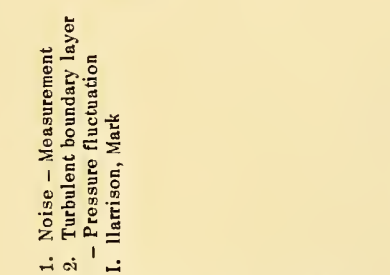 \\
\hline 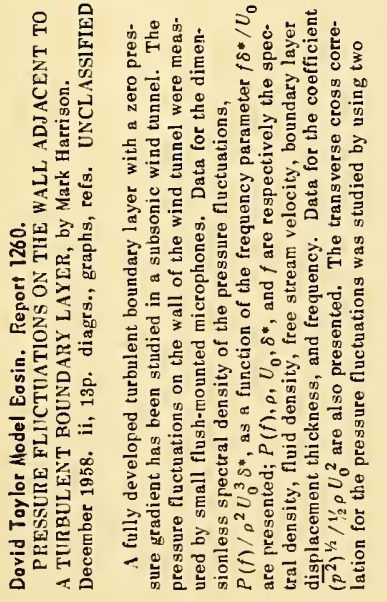 & 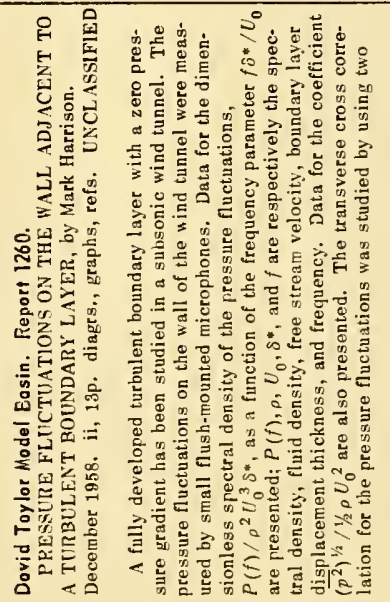 \\
\hline
\end{tabular}



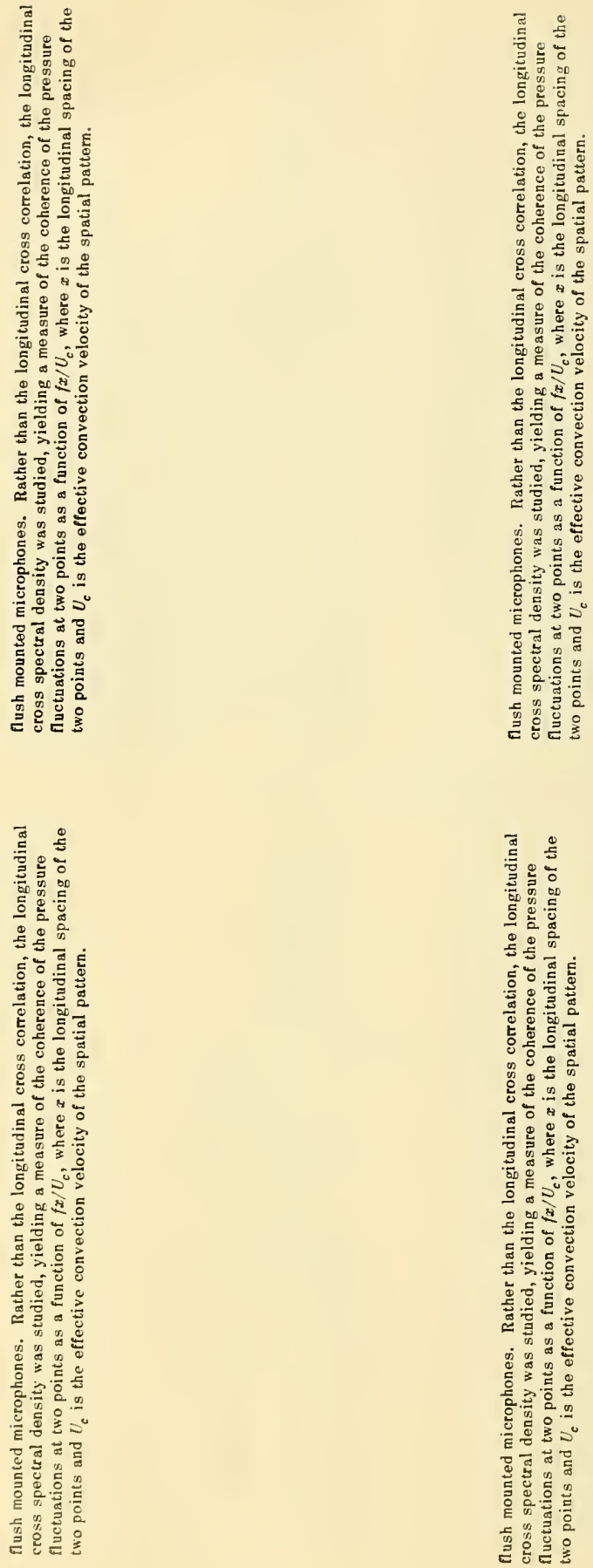
